\title{
Design and Implementation of Remote Health Monitoring Sensor Network based on the Internet of Things
}

\author{
Lihua Zhou ${ }^{\mathrm{a}}$, Qing Lai ${ }^{\mathrm{b}, *}$ \\ College of Medical Informatics, Chongqing Medical University, 1 Yixueyuan Road, Chongqing 400016, China \\ azhoulihuacq@gmail.com, blaiqingcmu@outlook.com \\ *Corresponding author
}

\begin{abstract}
From the view of practical application, this paper designs the human body physiology information collection nodes, the coordinator node, wireless transmission system using CC2430 microcontroller, and detailed design information of human body sensor (temperature, heart rate, ECG / pulse) circuit diagram. This paper set up software of a wireless network (ZigBee protocol), sensor signal acquisition, wireless transmitting and receiving, and communicate with PC machine by serial communication coordinator node. Experiments show that the network node data transmission accurate, reliable work, basically reached the requirements of the design issues.
\end{abstract}

Keywords-remote monitoring; CC2430; sensor node; coordinator node; zigbee protocol.

\section{INTRODUCTION}

Human physiological parameters is an important reference to measure the health status of people, carries on the real-time monitoring plays an important role in the diagnosis and diseases treatment. But the traditional monitoring instruments have large volume, complicated operation, the demanding environment and need to use the deep professional knowledge, which is not conducive to human physiological parameters monitoring. Many problems exist in the traditional ward inspection. Medical monitoring system in this study can be important physiological parameters real-time acquisition of the patient, the doctor can see patient information at any time through the management platform, when the patient is abnormal, the doctor can take timely measures to rescue by the alarm, so as not to delay treatment of the patient.

\section{THE DESIGN PRINCIPLE OF MEDICAL MONITORING SYSTEM}

The sensor node will be various medical sensors and integrated corresponding circuit, which can real-time collect temperature, heart rate, respiration, pulse, blood pressure, ECG and other physiological parameters, and wirelessly send the information to the routing node or directly sent to the coordinator node, the process should pay attention to the problem node coverage, the choice of transmission routing in the process. Coordinator node is responsible for gathering to routing node and the sensor node information, through the RS3232 serial port to send to the monitoring center, the doctor and the nurse on duty through the monitoring management center at any time to view the status of the patient physiological parameters, not only can be found in time of emergency that may occur in critically ill patients, can also provide the best advice and means of health care for the ordinary patients. Overall structure of medical monitoring system is as shown in Fig.1

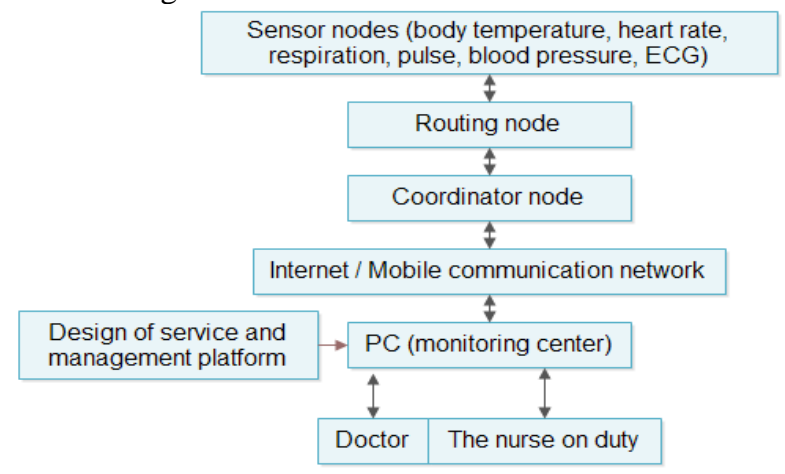

Figure 1. The structure diagram of health monitor system

Node is the basic functional unit of the wireless sensor networks, due to the specific application areas and different places, the design of the joint are not the same. Coordination are basically the same as the hardware device nodes and routing nodes, mainly include: processing module, communication module and power module. The sensor node consists of a sensor unit, signal processing unit, communication module and power supply module. The nodes of the structure shown in Fig.2. In addition, according to the requirements of specific application can also add other functional units, such as: positioning system, mobile system and power supply from the power supply system etc..

\begin{tabular}{|c|c|c|c|c|c|c|}
\hline \multirow{2}{*}{ Sensor } & \multirow{2}{*}{$\rightarrow A D C$} & $80 C 51$ & $\begin{array}{c}\text { Wireless transmit/ } \\
\text { receiver }\end{array}$ & $\begin{array}{l}\text { Wireless transmit/ } \\
\text { receiver }\end{array}$ & $80 C 51$ & $\begin{array}{c}\text { Serial } \\
\text { communication }\end{array}$ \\
\hline & & \multicolumn{2}{|r|}{ CC2430 } & \multicolumn{3}{|c|}{ CC2430 } \\
\hline 1 & $\uparrow$ & & $\uparrow$ & \multicolumn{3}{|c|}{ 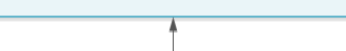 } \\
\hline \multicolumn{4}{|c|}{ Power module } & \multicolumn{3}{|c|}{ Power module } \\
\hline
\end{tabular}

Figure 2. The structure of wireless node 


\section{THE HARDWARE DESIGN OF MEDICAL MONITORING SYSTEM}

This paper uses the CC2430 chip, various medical sensors and wireless communication technology to complete the hardware design of medical monitoring system for real-time monitoring of human physiological information, including circuit design of sensor nodes, routing nodes and coordinator node hardware design block diagram, medical monitoring system as shown in Fig.3

Wireless communication module

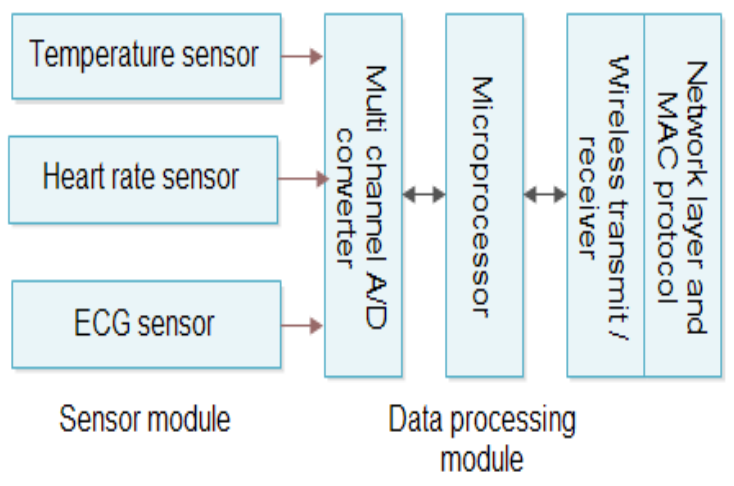

Figure 3. The hardware diagram of the medicine monitoring system

(1) The temperature measurement circuit

Temperature refers to the internal body temperature, is to understand the important index of life state, there is a close relationship between temperature and human body health, natural medicine has to maintain appropriate temperature of healthy view. According to the characteristics of human body temperature, temperature measurement range between 25 DEG -45 DEG C, the temperature resolution greater than $0.10 \mathrm{C}$ and other technical indicators, this design uses the DS digital temperature sensor 18B20. According to the working principle of DS 18B20, CC2430 only requires a single I/O interface line, the interface circuit is very simple, it is as shown in Fig.4. Because the DS 18B20 data output is an open drain output, data terminal must be connected to the 4.7K pullup resistor.

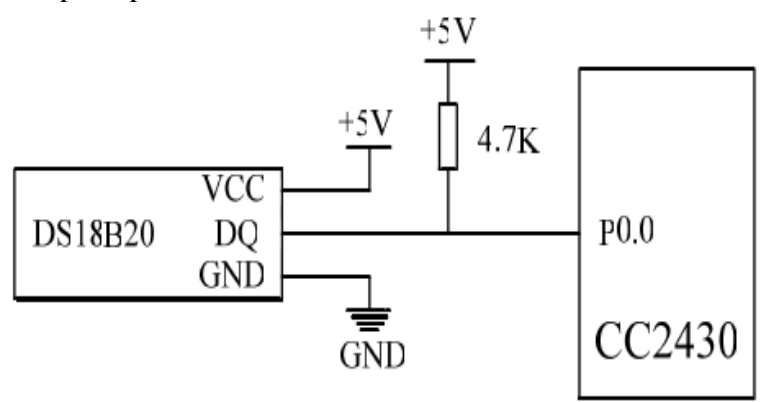

Figure 4. The schematic diagram of connection between cc2430 and ds18b20

(2) The principle of infrared pulse measurement
In the heart is beating, the human blood is most adequate; when heart contract, blood flow of human tissue has little flow. Infrared light irradiate onto the finger by the infrared emission diode that can rapidly absorb infrared light by finger blood tissue. When blood flow is sufficient attenuation, small attenuation less blood flow. Then, using the infrared receiving diode to receive finger transmission over the infrared light, larger infrared light represent received contraction of the heart, lower infrared light express beating heart. According to the human heart periodic beating, infrared intensity infrared receiving diode can receive periodical change of output voltage signal, it reflects the process of beating heart.

From the original signal waveform can see the whole change trend of pulse wave, if we want to get the available signal of pulse $0.05 \mathrm{HZ}-10 \mathrm{HZ}$, the key is to eliminate all kinds of interference, pulse signal acquisition of the sensor to the shaping and filtering and amplification processing. The circuit shown in Fig.5

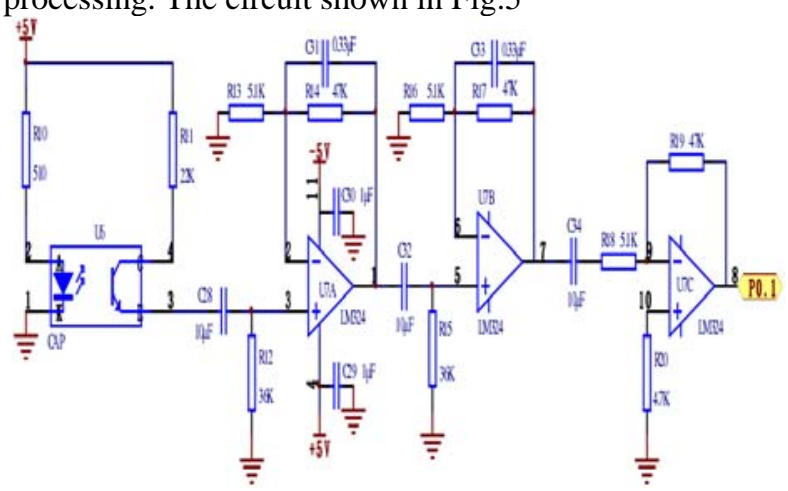

Figure 5. Pulse wave collecting circuit

(3) The design of ECG measurement circuit

ECG signal is an important signal of human body monitoring, ECG signal is the electrical activity of the heart that comprehensive reflect on the surface of the body, has an important significance for the diagnosis of heart disease ECG, commonly used in clinical disease diagnosis as an auxiliary means. The electrocardiogram is a reflection of excited process of the heart electrical activity, the basic function of the heart and its pathological study has an important reference value. Circuit design is shown in Fig.6

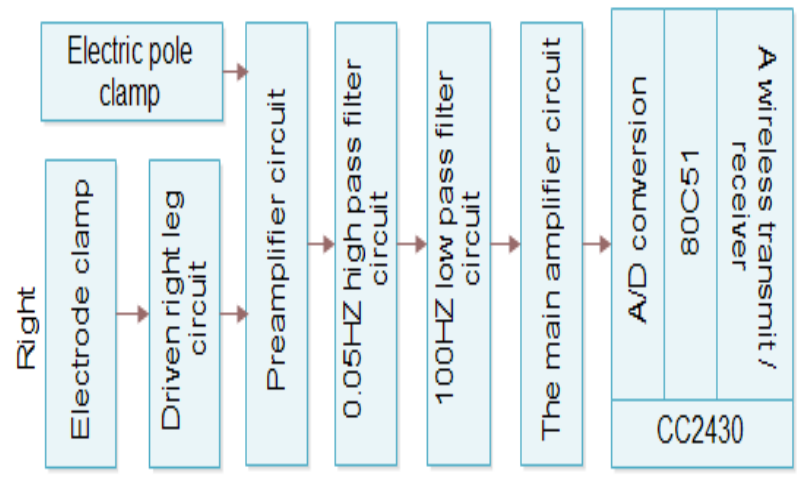

Figure 6. The circuit block diagram of ECG acquisition 


\section{DESIGN OF MONITORING SYSTEM SOFTWARE}

There are mainly three kinds of node monitoring network, which is sensor nodes, routing nodes and coordinator node. Coordination node responsible for the establishment and initialization of network, transmission network beacon, determining the channel network work and assigned 16 bit network address after electric starting [4]. Routing nodes joining the network is responsible for receiving the sensor node data and the data is transmitted to the coordinator node. Sensor nodes only transmit and receive signals, forwarding function does not have the power to start automatically after the launch, the binding request, to be with the father (routing) node establishes a binding, the collected body temperature, pulse and ECG data send routing node. In addition, the coordinator node sends the data to PC through serial port, the doctor can make data query and analysis through the user management interface. Network flow is as shown in Fig.7
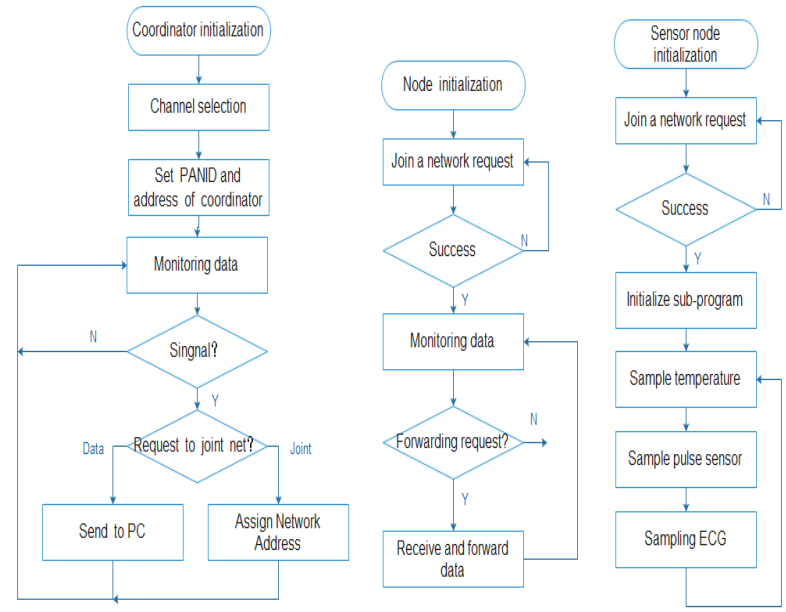

Figure 7. Network flow chart of CC2430

\section{CONCLUSION}

This paper designed a real-time monitoring inpatient physiological signal system, focusing on the completion of the design of hardware circuit and corresponding software program design of sensor nodes, the coordinator node. Among them, the sensor nodes can collect temperature, pulse and ECG of three physiological signals, together with the routing nodes, the coordinator node is composed of wireless network, and the collected physiological information is transmitted to the host computer information management system for doctors to see at any time.

\section{ACKNOWLEDGEMENTS}

This paper Supported by the project funds of Chonngqing Yuzhong District Technology Plan, Project Number: 20110405

\section{REFERENCES}

[1] Zhu Jianxin, high Reina, Zhang Xinfang. The health system of wearable pervasive Era [J]. Computer application system, 2008,10:5.

[2] Zhao Ze, Cui Li. A remote health care system based on Wireless Sensor Network [J]. information and control, 2006,4:265.

[3] Zheng Jiewen. Mobile health monitoring system of wearable real-time diagnosis, alarm [D]. Beijing: China people's Liberation Army Military Medical Science Academy of the PLA, 2008

[4] Li Haomin. Research on multi parameter remote monitoring system and its key technology [J], Physiological 2008,04.

[5] He Dayu, Wei iridium, Xu Yingpeng. Medical ZigBee wireless sensor network application research [J]. Journal of Changchun University of Technology (NATURAL SCIENCE EDITION), 2010,06:355-359. 